\title{
Effectiveness of 8-Week Glecaprevir/Pibrentasvir for Treatment-Naïve, Compensated Cirrhotic Patients with Chronic Hepatitis C Infection
}

\author{
Steven L. Flamm · Jens Kort - Steven E. Marx · John Strezewski • \\ Douglas E. Dylla · Bruce Bacon · Michael P. Curry · Naoky Tsai • \\ Nicole Wick
}

Received: January 17, 2020 / Published online: April 11, 2020

(C) The Author(s) 2020

\section{ABSTRACT}

Introduction: Glecaprevir/pibrentasvir (G/P) was approved on 26 September 2019 by the US Food and Drug Administration for 8-week duration in treatment-naïve (TN) hepatitis $\mathrm{C}$ virus (HCV)-infected patients with compensated cirrhosis (CC). Evidence from the EXPEDITION-8 study demonstrated that 8 weeks of G/P achieved a 98\% intent-to-treat (ITT) sustained virologic response rate 12 weeks post treatment (SVR12) in $343 \mathrm{TN} / \mathrm{CC}$ patients. The

Enhanced Digital Features To view digital features for this article go to https://doi.org/10.6084/m9.figshare. 11974200.

\section{S. L. Flamm}

Northwestern University Feinberg School of

Medicine, Chicago, IL, USA

J. Kort · S. E. Marx $(\varangle) \cdot$ J. Strezewski · D. E. Dylla

AbbVie, Mettawa, IL, USA

e-mail: steve.marx@abbvie.com

B. Bacon

Saint Louis University School of Medicine, St. Louis, MO, USA

M. P. Curry

Beth Israel Deaconess Medical Center, Boston, MA, USA

N. Tsai

University of Hawaii, Honolulu, HI, USA

N. Wick

Trio Health Analytics, La Jolla, CA, USA aim of this study is to demonstrate the first US real-world effectiveness of G/P 8-week treatment in genotype 1-6 TN/CC HCV patients.

Methods: Data from 73 TN/CC patients who initiated 8 weeks of $G / P$ treatment between August 2017 and November 2018 were collected electronically from providers and specialty pharmacies of the Trio Health network and analyzed. Cirrhosis was determined by FIB-4 $>5.2$ or was physician reported. The primary outcome was Per Protocol (PP) SVR12.

Results: The majority (60\%) of patients were male, with (mean values): age 59 years, body mass index (BMI) of 30, aspartate aminotransferase (AST) 105, and alanine aminotransferase (ALT) $101 \mathrm{IU} / \mathrm{ml}$. HCV genotypes (GT) were: GT1 81\% (59/73), GT2 10\% (7/73), GT3 5\% (4/ 73), GT4 3\% (2/73), and GT6 1\% (1/73). Eight percent $(6 / 73)$ of patients had concurrent proton pump inhibitor (PPI) use, and 15\% (11/72) had a baseline viral load $>6 \mathrm{MM} \mathrm{IU} / \mathrm{ml}$. Zero patients discontinued, two patients were reported as lost to follow-up, and there was one virologic failure. PP sustained virologic response at 12 weeks (SVR12) rate was 99\% (70/71), and the intent-to-treat (ITT) SVR12 rate was 96\% (70/73).

Conclusions: Early real-world experience indicates high effectiveness of the 8-week G/P regimen in a diverse treatment-naïve, compensated cirrhotic US population. 
Keywords: 8-weeks; Glecaprevir/pibrentasvir; Hepatitis C virus; Infectious diseases; Treatment naïve

\section{Key Summary Points}

Why carry out this study?

Expedition- 8 is the first phase $3 \mathrm{~b}$ trial to demonstrate a 98\% (335/343) intent-totreat (ITT) sustained virologic response rate 12 weeks post treatment (SVR12) in 343 treatment-naïve (TN) compensated cirrhosis (CC) patients after 8 weeks of glecaprevir/pibrentasvir (G/P).

Subsequently, G/P was approved on 26 September 2019 by the US Food and Drug Administration for an 8-week duration in TN hepatitis $\mathrm{C}$ virus (HCV)-infected patients with CC.

To date, there are no published US realworld studies of $\mathrm{G} / \mathrm{P}$ with 8-week duration in TN/CC HCV patients.

\section{What was learned from this study?}

Early real-world experience indicates high effectiveness of an 8-week G/P regimen in a diverse treatment-naïve, compensated cirrhotic US population.

Results showed a high SVR12 rate of 96\% (70/73 by ITT analysis) and $99 \%$ [70/71 by per protocol (PP) analysis], similar to the results of the EXPEDITION-8 registration study.

\section{INTRODUCTION}

The National Health and Nutrition Examination Survey (NHANES) estimated that there are 2.4 million hepatitis $\mathrm{C}$ virus (HCV) patients in the USA [1]. Chronic HCV infection is a leading cause of liver fibrosis that may progress to liver cirrhosis, hepatocellular carcinoma or hepatic decompensation. Hepatitis $\mathrm{C}$ is the leading cause of death in the US compared with the next 60 reportable chronic infectious diseases combined in 2013 [2]. Patients living with HCV exhibit significantly greater risks for extra-hepatic manifestations and higher medical costs. Treatment of hepatitis C significantly mitigates this economic burden from HCV/hepatic complications and extrahepatic manifestations [3]. However, only about $19 \%$ of the HCV ribonucleic acid (RNA)-positive patients were treated for HCV in the US in 2016 [4]. While eliminating HCV may be extremely challenging, the World Health Organization (WHO) and multiple public policy advocacy groups have established goals to eliminate HCV by 2030 [5].

Glecaprevir/pibrentasvir (G/P) was initially approved in August 2017 for an 8-week duration in treatment-naïve (TN) non-cirrhotic (NC) patients with chronic $\mathrm{HCV}$ infection and for a 12-week duration in TN-compensated cirrhotic (CC) patients across genotypes (GT) 1-6. In September 2019, the US Food and Drug Administration (USFDA) approved a shorter 8-week treatment duration for G/P in GT 1-6 TN CC patients. FDA approval was based on the EXPEDITION-8 trial, which demonstrated 98\% sustained virologic response at 12 weeks post treatment (SVR12) in 343 TN/CC patients with chronic HCV GT 1-6 infection [6]. A previous real-world study in $560 \mathrm{HCV}$ TN/NC patients in the US with GT 1-6 infection demonstrated similar efficacy of an 8-week G/P treatment duration compared with registration trials [7]. The aim of this study is to provide the first US real-world effectiveness data of G/P 8-week treatment duration in TN/CC HCV patients.

\section{METHODS}

Real-world data from 73 TN/CC patients who initiated 8 weeks of $G / P$ treatment between August 2017 and November 2018 were collected electronically from providers and specialty pharmacies of the Trio Health network and analyzed. The Trio Health network is a platform designed as a portal for specialty pharmacies and physicians to collaborate on efforts to improve patient care. Data were entered into the TRIO Health's proprietary Innervation 
Platform through nightly file feeds and manual user data entry. This portal provides applied proprietary logic to identify and prompt users of input errors and missing data. Where mandated by state law, patients signed an electronic informed consent allowing specialty pharmacies to collect the data. Each participating clinician and specialty pharmacy signs an agreement attesting to compliance with all rules and regulations regarding the disclosure of patient information to a shared prescription database. FDA-mandated reporting of deaths and/or adverse events was assumed to be completed at the time of the event. The causes and outcomes of individual events were not known. TRIO Health Analytics was provided with deidentified, HIPAA-compliant patient information from the platform. A letter of exemption from the Western Institutional Review Board was received since this is a retrospective review of existing data recorded by the investigator in such a manner that the identity of the human subjects cannot readily be ascertained directly or through identifiers linked to the subjects, the investigator does not contact the subjects, and the investigator will not re-identify subjects. The primary outcome measure in this study is an HCV plasma RNA below the limit of quantification obtained 12 weeks after the end of G/P treatment (SVR12). A window of 10-26 weeks post treatment was acceptable for the primary outcome measure. The primary end point of this study is the per protocol (PP) SVR12 rate in GT1-6 TN/CC HCV patients defined as adult patients who have received 8 weeks of G/P therapy and with an HCV RNA viral load measurement $10-26$ weeks post treatment. The secondary end point is the intent-to-treat (ITT) SVR12 rate defined as all adult patients who initiated 8 weeks of G/P therapy.

Patients were included if they were TN/CC, $>$ 18 years of age, initiated 8 -week G/P (drug dispensed to patient), genotype $1-6$, and had sufficient follow-up time in the database to obtain an SVR12 assessment. Patients were excluded if they were currently enrolled in a clinical trial; received initial prescription for $>8$ weeks of G/P; had decompensated cirrhosis; current hepatocellular carcinoma (HCC) (previously treated HCC was permitted); had prior HCV treatment; had missing age, missing fibrosis staging, missing prior HCV treatment status, missing or mixed genotype; and/or had a liver transplant.

Treatment-naïve was defined as no prior $\mathrm{HCV}$ treatment, reported by the treating physician. Cirrhosis was defined as a FIB-4 score $>5.2$ [4] or was physician reported. Decompensated cirrhosis was defined as no current or past clinical evidence of Child-Pugh B or C classification or clinical history of liver decompensation including ascites, bleeding esophageal varices, or hepatic encephalopathy. Compensated cirrhosis was defined as cirrhosis with no prior history of liver decompensation.

Virologic failures were physician reported and defined as the first HCV viral load anytime post G/P 8-week treatment where the provider indicates a detectable level of HCV or the actual measure is above the lower level of quantification. Treatment start and end dates are defined by pharmacy dispensing records.

Baseline demographic and clinical characteristics consisted of information obtained closest to the start of therapy. These included: age, ethnicity, body mass index (BMI), and gender; laboratory values including alanine aminotransferase (ALT), aspartate aminotransferase (AST), platelets, hemoglobin, serum creatinine, estimated glomerular filtration rate (eGFR), and hepatitis $\mathrm{C}$ virus ribonucleic acid (HCV RNA); comorbidities including human immunodeficiency virus (HIV), hepatitis B virus (HBV), anxiety, depression, chronic kidney disease (CKD), hyperlipidemia, and hypertension; genotype/subtype; concurrent proton pump inhibitor (PPI) use during $\mathrm{HCV}$ treatment; physician practice site; payer type (commercial, Medicare, Medicaid, other); and practice type (academic, community). Academic centers are defined as tertiary care centers or clinics affiliated with academic centers. Any other practice (solo or group) is characterized as a community site.

For patient disposition, lost to follow-up is defined as patients who were unreachable either during or after completion of treatment. SVR12 rates are calculated by dividing the number of HCV patients that achieved an SVR12 by the total number of patients in the study. 


\section{RESULTS}

The majority (60\%) of HCV patients were male, with a mean age of 59 years of age, BMI of 30 , AST 105, and ALT $101 \mathrm{IU} / \mathrm{ml}$. HCV genotypes (GT) were: GT1 81\% (59/73), GT2 10\% (7/73), GT3 5\% (4/73), GT4 3\% (2/73), and GT6 1\% (1/ $73)$. Eight percent $(6 / 73)$ of patients had concurrent PPI use, and 15\% (11/72) had a baseline viral load $>6$ million international units (IU)/ milliliter (ml) (Table 1). No patients discontinued G/P therapy, two patients were reported as lost to follow-up, and there was one virologic failure (Fig. 1). The PP SVR12 rate was 99\% (70/ $71)$, and the ITT SVR12 rate was 96\% (70/73) (Table 2). The two patients that were lost to follow-up were both from community practices. One was a 65-year-old male with GT1b infection, $820,000 \mathrm{IU} / \mathrm{ml}$ baseline viral load, and concomitant PPI use. The second patient was a 73-year-old female with GT1 infection, $1,600,000 \mathrm{IU} / \mathrm{ml}$ baseline viral load. One patient with virologic failure relapsed after treatment. This patient was a male, 51 years old, with GT6 infection and a baseline viral load of $50,000,000 \mathrm{IU} / \mathrm{ml}$, on Medicaid, and from a community practice (Table 3 ).

\section{DISCUSSION}

G/P was recently approved by the US Food and Drug Administration for use with a shortened treatment duration of 8 weeks in TN/CC patients with chronic HCV genotype 1-6 infection [8]. Here we report the first real-world effectiveness data of G/P 8-week treatment in HCV TN/CC patients from the US TRIO health community and academic healthcare providers who choose to utilize an 8-week treatment duration for TN/CC patients prior to FDA approval of the 8-week treatment duration. There are very limited ex-US real-world data on the 8-week effectiveness of glecaprevir/pibrentasvir in TN/CC HCV patients, and none of these studies have assessed more than ten HCV TN/CC patients [9-11]. In this limited sample, our results showed a high SVR12 rate of $96 \%$ (70/73 by ITT analysis) and 99\% (70/71 by PP analysis), similar to the results of the
Table 1 Demographics and baseline characteristics

\begin{tabular}{|c|c|}
\hline Characteristics & $n /$ total $(\%)$ \\
\hline Community practice & $63 / 73(86)$ \\
\hline \multicolumn{2}{|l|}{ Payer type } \\
\hline Commercial & $19 / 73(26)$ \\
\hline Medicaid & $22 / 73(30)$ \\
\hline Medicare & $31 / 73(42)$ \\
\hline Other & $1 / 73(1)$ \\
\hline Male & $44 / 73(60)$ \\
\hline BMI, median (range) & $30(19-53)$ \\
\hline Age, median (range) & $59(31-76)$ \\
\hline \multicolumn{2}{|l|}{ Ethnicity } \\
\hline White & $20 / 73(27)$ \\
\hline Black & $7 / 73(10)$ \\
\hline Asian & $2 / 73(3)$ \\
\hline Hispanic or Latinx & $4 / 73(5)$ \\
\hline Other or unspecified & $40 / 73(55)$ \\
\hline Concurrent PPI use & $6 / 73(8)$ \\
\hline \multicolumn{2}{|l|}{ Chronic kidney disease stage } \\
\hline No CKD & $40 / 67(61)$ \\
\hline $1-3$ & $25 / 67(37)$ \\
\hline $4-5$ & $1 / 67(1)$ \\
\hline \multicolumn{2}{|l|}{ Comorbidities } \\
\hline Anxiety & $12 / 73(16)$ \\
\hline Depression & $13 / 73(18)$ \\
\hline Diabetes & $12 / 73(16)$ \\
\hline Hypertension & $33 / 73(45)$ \\
\hline Hyperlipidemia & $5 / 73(7)$ \\
\hline HIV co-infection & $1 / 73(1)$ \\
\hline HBV co-infection & $2 / 73(3)$ \\
\hline \multicolumn{2}{|l|}{ Baseline laboratory } \\
\hline ALT U/l, mean $(\mathrm{SD}) n=73$ & $101(125)$ \\
\hline AST U/l, mean $(\mathrm{SD}) n=70$ & $105(184)$ \\
\hline $\mathrm{eGFR}<30 \mathrm{ml} / \mathrm{min}$ & $1 / 67(1)$ \\
\hline $\mathrm{HCV}$ RNA $>6,000,000 \mathrm{IU} / \mathrm{ml}$ & $11 / 73(15)$ \\
\hline
\end{tabular}


Table 1 continued

\begin{tabular}{lc}
\hline Characteristics & $n /$ total (\%) \\
\hline HCV genotype & \\
1a & $41 / 73(56)$ \\
$1 \mathrm{~b}$ & $16 / 73(22)$ \\
1 (subtype unspecified) & $2 / 73(3)$ \\
2 & $7 / 73(10)$ \\
3 & $4 / 73(5)$ \\
4 & $2 / 73(3)$ \\
6 & $1 / 73(1)$ \\
\hline
\end{tabular}

EXPEDITION-8 registration study with an ITT SVR12 rate of $98 \%(335 / 343)$ and PP SVR12 rate of 99.7\% (334/335) [6]. Real-world evidence in addition to clinical trial results provides additional confidence and insights for healthcare providers. G/P is now the first and only 8 -week treatment approved for GT1-6 chronic HCV infection in treatment-naïve patients without cirrhosis, or with compensated cirrhosis. This shorter duration offers an added benefit of simplifying treatment and has the potential to reduce costs, which will be important as the world moves towards eliminating HCV. Epidemiology estimates of the US HCV population suggest that as many as $98 \%$ of all TN HCV patients could be treated with an 8-week regimen [12].

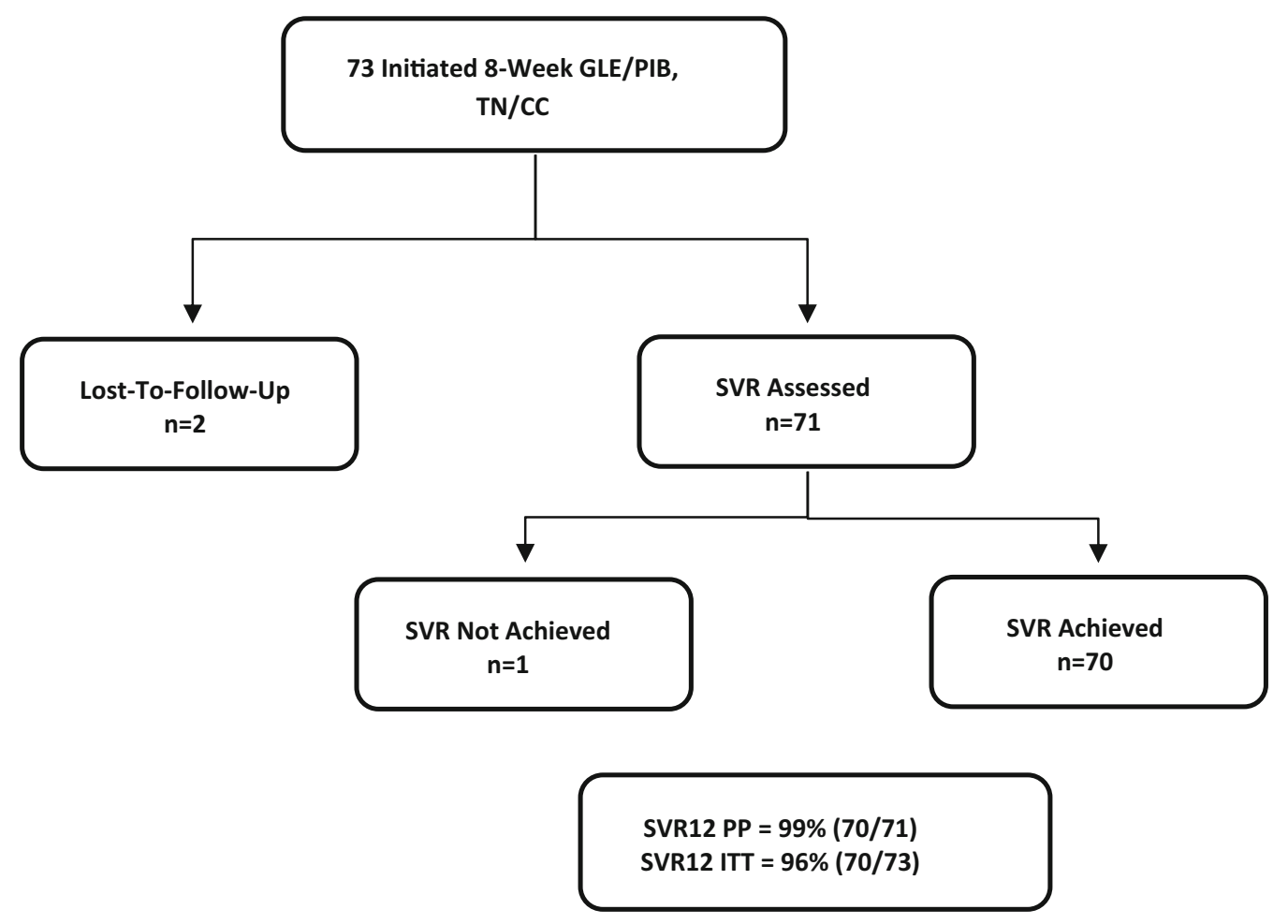

Fig. $1 \mathrm{HCV}$ patient disposition

Table 2 SVR12 rates by genotype

\begin{tabular}{lllllll}
\hline Genotype & GT1 & GT2 & GT3 & GT4 & GT6 & Total \\
\hline SVR12 PP & $100 \%(57 / 57)$ & $100 \%(7 / 7)$ & $100 \%(4 / 4)$ & $100 \%(2 / 2)$ & $0 \%(0 / 1)$ & $99 \%(70 / 71)$ \\
SVR12 ITT & $97 \%(57 / 59)$ & $100 \%(7 / 7)$ & $100 \%(4 / 4)$ & $100 \%(2 / 2)$ & $0 \%(0 / 1)$ & $96 \%(70 / 73)$ \\
\hline
\end{tabular}




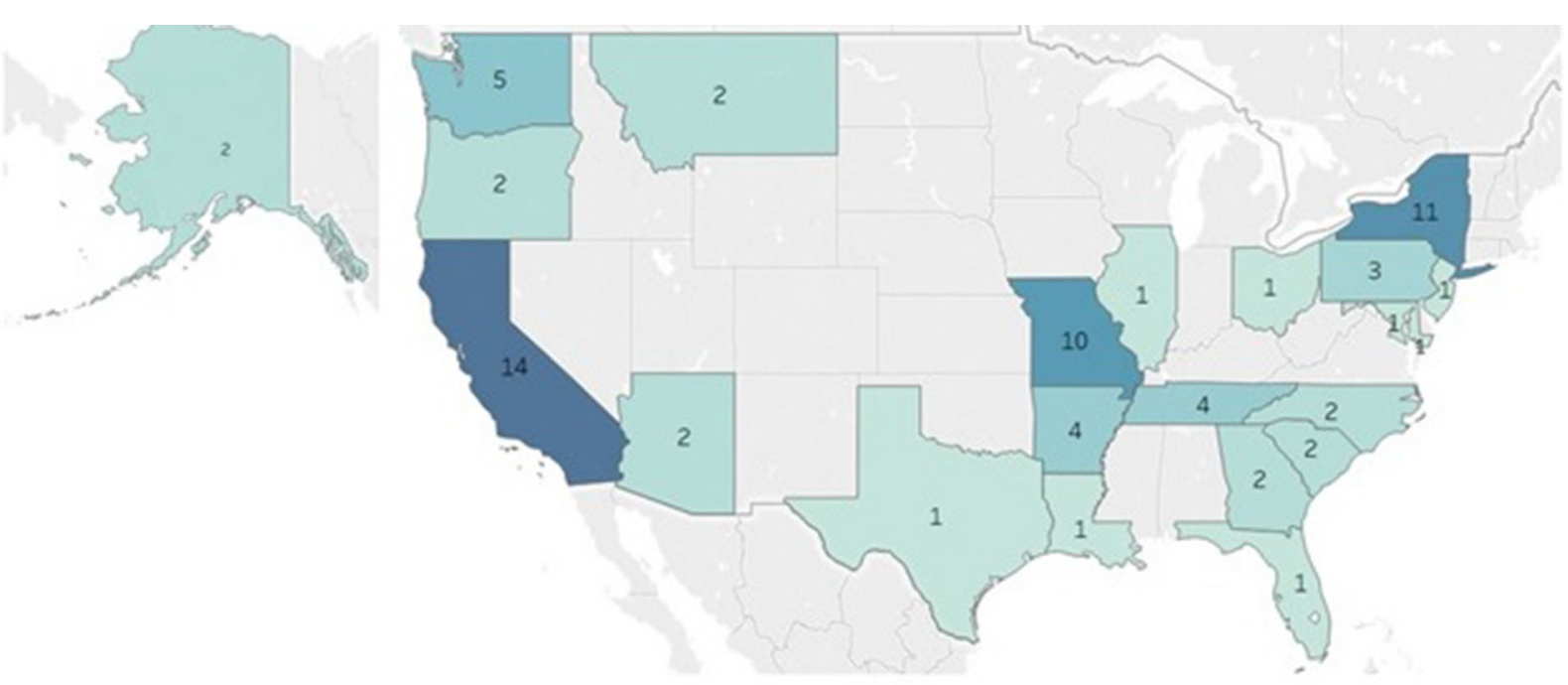

Fig. 2 Number of HCV patients, distribution by state

Table 3 Lost to follow-up and SVR12 not achieved patient characteristics

\begin{tabular}{|c|c|c|c|c|c|c|c|}
\hline Type & Patient & $\begin{array}{l}\text { Baseline } \\
\text { VL }\end{array}$ & Genotype & Comorbidities & $\begin{array}{l}\text { Concurrent } \\
\text { PPI }\end{array}$ & Insurance & $\begin{array}{l}\text { Practice } \\
\text { type }\end{array}$ \\
\hline Lost to follow-up & $65, \mathrm{M}$ & 820,000 & GT1B & None $^{b}$ & Yes & Commercial & Community \\
\hline Lost to follow-up & $73, \mathrm{~F}$ & $1,600,000$ & $\mathrm{GT} 1^{\mathrm{a}}$ & CKD stage 2 & No & Medicare & Community \\
\hline $\begin{array}{c}\text { SVR12 not } \\
\text { achieved }\end{array}$ & $51, \mathrm{M}$ & $50,000,000$ & GT6 & None $^{b}$ & No & Medicaid & Community \\
\hline
\end{tabular}

a Subtype mixed or unknown

b Collected comorbidities include: anxiety, depression, hyperlipidemia, hypertension, diabetes, HIV, HBV, CKD stage

Limitations of this retrospective observational study include that the study subjects who met the study criteria were included regardless of demographics. While the data include 22 states, these results may not be generalizable to the entire US population (Fig. 2). To further inform this analysis, a complete characterization of the sample population is included in Table 1. As with all real-world studies, results may be further confounded by factors not included as covariates. Recall bias was limited to provider input into patients' charts and to patients' recollection. Therefore, the data are reflective of information recorded by the physician and would only be flawed if recorded improperly at the practice. Recall bias was not applicable to the prescription or dispensing data, as it reflects actual transactions that occur at the pharmacy. As patients with identified decompensated Child-Pugh B or C cirrhosis are contraindicated for treatment with $G / P$, these patients were expressly excluded from this analysis based on a current or prior history of hepatic decompensation. Patient cirrhosis status included in the study was physician reported. While a bivariate analysis to identify associations of different variables with the outcome were planned, this was not possible, since only a single patient experienced a virologic failure.

The World Health Organization has established targets for HCV elimination by 2030; however, current rates of $\mathrm{HCV}$ treatment have been declining in the US, which can be 
attributed to lack of patient awareness of infection as well as access barriers to treatment. To help address patient barriers to HCV treatment, the US Preventive Services Task Force and Center for Disease Control have published draft recommendations that now calls for universal screening for HCV in adults [13, 14]. Prevention can reduce the rate of new infections, as well as deaths. The availability of a simple, 8-week HCV treatment regimen that can be utilized in the broadest population with HCV infection provides a shorter time to cure and additional opportunity to encourage untreated patients to initiate HCV therapy.

Given the similar high cure rates and safety profiles of contemporary $\mathrm{HCV}$ regimens, $\mathrm{HCV}$ patients' preferences for therapy may be influenced by treatment attributes other than cure rates and tolerability. Treatments that are more convenient and require less disruption to their daily life (e.g., shorter treatment duration, no modification in PPI use, and fewer office visits when on treatment) are important to patients with HCV and should be considered when making treatment decisions [15].

\section{CONCLUSIONS}

Early real-world experience from the TRIO health network indicates high effectiveness of an 8-week G/P treatment duration for chronic HCV infection in a diverse treatment-naïve US population with compensated cirrhosis.

\section{ACKNOWLEDGEMENTS}

Funding. The design, study conduct, and financial support for the study were provided by AbbVie. AbbVie funded the journal's Rapid Service and Open Access Fees.

Authorship. All named authors meet the International Committee of Medical Journal Editors (ICMJE) criteria for authorship for this article, take responsibility for the integrity of the work as a whole, and have given their approval for this version to be published.
Authorship Contributions. AbbVie participated in the interpretation of data, review, and approval of the manuscript. All authors vouch for the accuracy and completeness of the reported data and the adherence of the study to the protocol. All authors independently made the decision to submit the manuscript for publication.

Disclosures. Dr. Steven Flamm advises for, is on the speakers' bureau for, and received grants from Gilead Sciences Inc., and AbbVie Inc., and advises for Merck \& Co., Inc. Jens Kort, Steve Marx, John Strezewski, and Douglas Dylla are employees of AbbVie Inc. and may own company stock. Dr. Bruce Bacon consults for, is on the speakers' bureau for, and received grants from Merck \& Co. Inc. He advises for, is on the speakers' bureau for, and received grants from AbbVie Inc. and Gilead Sciences, Inc. He advises for and is on the speakers' bureau for Janssen Pharmaceuticals. He advises for and received grants from Bristol-Myers Squibb. He is on the speakers' bureau for Bausch Health Companies Inc. Dr. Michael Curry consults for and received grants from Gilead Sciences, Inc. He consults for Trio Health, Gilead Sciences Inc., AbbVie Inc., and Bristol-Myers Squibb. Dr. Naoky Tsai consults for, advises for, is on the speakers' bureau for, and received grants from Gilead Sciences Inc., Intercept Pharmaceuticals. Nicole Wick is employed by Trio Health and received grants from Gilead Sciences Inc., AbbVie Inc., and Merck \& Co., Inc.

Compliance with Ethics Guidelines. A letter of exemption from Western Institutional Review Board was received since this is a retrospective review of existing data that are recorded by the investigator in such a manner that the identity of the human subjects cannot readily be ascertained directly or through identifiers linked to the subjects, the investigator does not contact the subjects, and the investigator will not re-identify subjects.

Data Availability. The datasets generated during and/or analyzed during the current study are not publicly available because Trio Health Analytics' data collection platform 
conforms with HIPPA regulations; however, data may be available from Trio Health Analytics on reasonable request.

Open Access. This article is licensed under a Creative Commons Attribution-NonCommercial 4.0 International License, which permits any non-commercial use, sharing, adaptation, distribution and reproduction in any medium or format, as long as you give appropriate credit to the original author(s) and the source, provide a link to the Creative Commons licence, and indicate if changes were made. The images or other third party material in this article are included in the article's Creative Commons licence, unless indicated otherwise in a credit line to the material. If material is not included in the article's Creative Commons licence and your intended use is not permitted by statutory regulation or exceeds the permitted use, you will need to obtain permission directly from the copyright holder. To view a copy of this licence, visit http://creativecommons.org/licenses/by$\mathrm{nc} / 4.0 /$.

\section{REFERENCES}

1. Hofmeister MG, Rosenthal EM, Barker LK, et al. Estimating prevalence of hepatitis $C$ virus infection in the United States, 2013-2016. Hepatology. 2019;69:1020-31.

2. https://www.cdc.gov/nchhstp/newsroom/2016/HCVmortality.html. Accessed Oct 8, 2019.

3. Reau N, Vekeman F, Wu E, Bao Y, Gonzalez YS. Prevalence and economic burden of extrahepatic manifestations of hepatitis $\mathrm{C}$ virus are underestimated but can be improved with therapy. Hepatol Commun. 2017;1:439-52.

4. Chirikov VV, Marx SE, Manthena AR, Strezewski JP, Saab S. Development of a comprehensive dataset of hepatitis $\mathrm{C}$ patients and examination of disease epidemiology in the United States, 2013-2016. Adv Ther. 2018;35(7):1087-11024.
5. Waheed Y, Siddiq M, Jamil Z, Najmi MH. Hepatitis elimination by 2030: progress and challenges. World J Gastroenterol. 2018;24(44):4959-61.

6. Brown RS, Buti M, Rodrigues L, Chulanov V, et al. Glecaprevir/pibrentasvir for 8 weeks in treatmentnaïve patients with chronic HCV genotypes 1-6 and compensated cirrhosis: the EXPEDITION-8 trial (published online). J Hepatol 2019.

7. Flamm SL, Kort J, Marx S, et al. Effectiveness of 8-week glecaprevir/pibrentasvir (G/P) for treatment naïve, non-cirrhotic patients with $\mathrm{HCV}$ infection in the trio health network. Hepatology. 2018;68(1(suppl)):375A-6A.

8. https://www.fda.gov/news-events/press-announce ments/fda-approves-treatment-adults-and-childrenall-genotypes-hepatitis-c-and-compensated-cirrhosis. Accessed Oct 9, 2019.

9. D’Ambrosio R, Pasulo L, Puoti M, Vinci M, et al. Real-world effectiveness and safety of glecaprevir/ pibrentasvir in 723 patients with chronic hepatitis C. J Hepat. 2018;70:379-87.

10. Berg T, Nauman U, Stoehr A, Sick C, et al. Realworld effectiveness and safety of glecaprevir/pibrentasvir for the treatment of chronic hepatitis $\mathrm{C}$ infection: data from the German Hepatitis C-Registry. APT. 2019;49:1052-9.

11. Ogawa E, Furusyo N, Nakamuta M, Nomura H, et al. Glecaprevir and pibrentasvir for Japanese patients with chronic hepatitis $C$ genotype 1 or 2 infection: results from a multicenter, real-world cohort study. Hepatol Res. 2019;49:617-26.

12. Data on file. AbbVie Inc. Ipsos Healthcare HCV USA Therapy Monitor (July 16, 2018-March 2019, all data collected online) (CIpsos 2019, all rights reserved.

13. https://www.uspreventiveservicestaskforce.org/Page/ Document/draft-recommendation-statement/hepati tis-c-screening1. Accessed Oct 8, 2019.

14. https://www.regulations.gov/document?D=CDC2019-0094-0001. Accessed Nov 21, 2019.

15. Welzel TM, Yang M, Sajeev G, et al. Assessing patient preferences for treatment decisions for new direct acting antiviral (DAA) therapies for chronic hepatitis C virus infections. Adv Ther. 2019;36(9): 2475-86. 